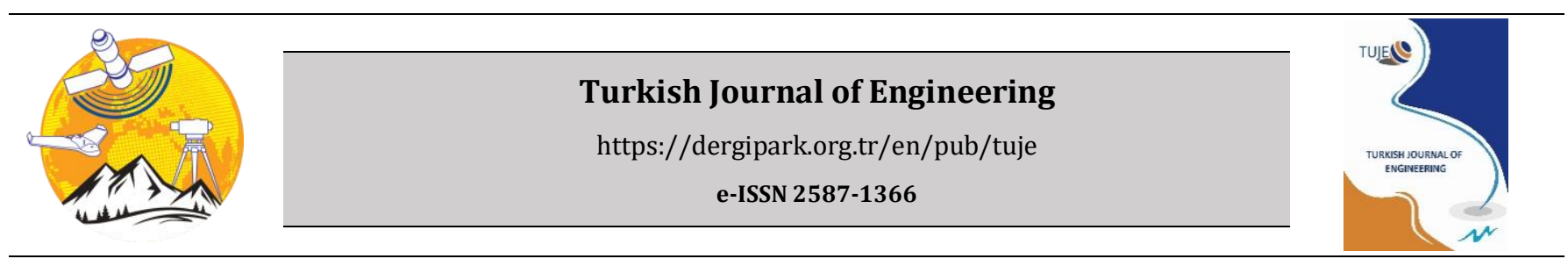

\title{
Performance evaluation of model predictive control method for neutral point clamped inverter
}

\author{
Ozan Gülbudak*1@, Mustafa Gökdağ 1 (1) \\ ${ }^{1}$ Karabuk University, College of Engineering, Electrical-Electronics Engineering, Karabük, Turkey
}

\section{Keywords}

Model predictive control

NPC inverter

Optimal control

Optimization

Power converters

\begin{abstract}
The neutral-point clamped (NPC) inverter is a popular three-level converter topology used in motor drive applications and other dc/ac converter systems. In this paper, the performance evaluation of the model predictive control is performed to investigate its applicability in controlling NPC inverters. The model predictive control (MPC) is a promising closed-loop control strategy in applications where multiple control goals are considered. The ease of adding the objectives to the control law improves the reputation of the MPC. The numerous control goals can be regulated in single feedback. Thus, the adequate bandwidth is noticeably higher compared to the traditional linear controllers. However, controlling the multiple objectives require the use of weighting factors to tune the system performance. Regarding the system performance, multiple reference tracking performance is investigated in this study. Our case study considers three control goals: output load current, switching frequency control, and capacitor voltage balancing. The predictive control is designed to regulate these dynamics, and comprehensive performance analyses are performed. The designed controller is tested using a simulation tool. The simulation results prove that predictive control offers a n excellent multi-objective control performance provided that the weighting factors and other design parameters are finely adjusted. The poor selection of the design parameters affects the closedloop performance, and the conducted analyses show the effects of the controller parameters.
\end{abstract}

\section{INTRODUCTION}

The neutral-point clamped (NPC) inverters are broadly preferred in ac drive applications and power electronics systems. It is a three-level three-phase inverter that can utilize three different voltage levels $(0.5 v d c, 0,-0.5 v d c)$ at the inverter output. This offers a substantial benefit regarding reducing the voltage stress on the active switches. Furthermore, the power level can be doubled due to the half voltage across switching devices. The other important aspect of NPC inverter is that the first few harmonics are centered around the two times higher of the operating frequency (switching frequency) (Klabunde et al. 1994; Nabae, et al. 1981; Rojas et al. 1995). This simplifies the procedure of selecting the filter parameter; thus, more efficient filtering capability is attained. A superior harmonics attenuation is achieved, improving the quality of the controlled variables, such as the output voltage or current. Besides the topological advantages of NPC inverters, a higher number of active switches is required to utilize the NPC inverter. In a traditional two-level voltage source inverter, only 6 active switches are used.

However, the NPC inverter employs the 12 active switches, and more active switches increase the switching losses. The other critical point is that the control complexity is higher compared to the two-level topologies. In particular, the capacitor voltage balancing must be considered in the controller design process. In the literature, the voltage balancing problem has been reported in several research papers (Jarutus and Kumsuwan, 2017). Different types of strategies have been investigated to overcome the capacitor voltage imbalance problem.

In the literature, different control and modulation strategies have been reported for NPC inverter systems. The optimal switching frequency technique was introduced in (Steinke, 1992). An analytical method to 
analyze NPC inverter dynamics and the performance of the capacitor voltage balancing are extensively examined (Ogasawara and Akagi, 1993). A comprehensive stability analysis of neutral-point control (Newton and Sumner, 1997) has been performed, a model that explains the system dynamics has been proposed. Moreover, a predictive control scheme has been introduced in (Vargas et al. 2007), multi-objective optimization problem has been formulated to control NPC inverter. The self-balancing effect has been investigated in (Jie et al. 2011), the effects of the self-balancing on the switching frequency have been reported. Another interesting work (Choi et al. 2015) shows that the voltage oscillations can be reduced by introducing an offset in time to the switch turn-on procedure. Other control mechanisms are also available in the literature. To gain a depth understanding of the NPC system, a good survey paper (Rodriguez et al. 2010) can be referred to.

In this study, the model predictive control method is investigated to analyze the dynamic characteristic of MPC. The control goals are considered the multiobjective optimization problem and the output load current, capacitor voltage balancing, and average switching frequency. The effects of the design parameters are examined, the feasibility of the MPC strategy in controlling NPC inverter is explored. The simulation works verify the system model and the formulated MPC controller. The simulations work demonstrates the potency of the MPC method; however, the design parameters have considerable influences on the steady-state and transient performance.

\section{SYSTEM MODEL}

In this section, the system mode of the NPC inverter is given. The NPC inverter topology schematic is illustrated in Fig. 1. Each NPC inverter leg has four active switches and two diodes; a high number of switching combinations are possible in this configuration. However, there are some switching restrictions to ensure stable operation. Thus, some of these switching states are not permissible. By considering the switching limitations, the NPC inverter yields 27 allowable switching states. To have a significant sub-set (solution set) allows flexibility in designing the objective function. In NPC inverter, the switching state $S_{j \alpha}$ refers to switching status of phase $j$, with $j \in\{a, b, c\}$ and $\alpha \in\{1,2,3,4\}$. At the output of the inverter stage, three discrete voltage levels can be generated. The switching state for each phase is summarized in Table 1 . The parameter $P$ refers to positive dc voltage, and $\mathrm{N}$ refers to negative dc voltage. 0 denotes the zero dc voltage at the output of the inverter stage.

Table 1. The switching states for each phase

\begin{tabular}{llllll}
\hline $\mathrm{S}_{\mathrm{j}}$ & $\mathrm{S}_{\mathrm{j} 1}$ & $\mathrm{~S}_{\mathrm{j} 1}$ & $\mathrm{~S}_{\mathrm{j} 1}$ & $\mathrm{~S}_{\mathrm{j} 1}$ & $\mathrm{~V}_{\mathrm{j} 0}$ \\
\hline $\mathrm{P}$ & 1 & 1 & 0 & 0 & $\mathrm{Vdc} / 2$ \\
0 & 0 & 1 & 1 & 0 & 0 \\
$\mathrm{~N}$ & 0 & 0 & 1 & 1 & $-\mathrm{V}_{\mathrm{dc}} / 2$ \\
\hline
\end{tabular}

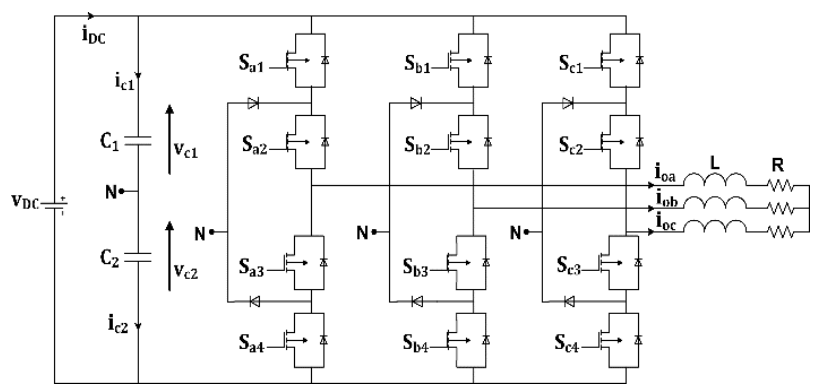

Figure 1. The neutral-point clamped inverter circuit diagram.

Some voltage vectors that the NPC inverter can produce are redundant. Thus, these voltage vectors can be eliminated in the controller design process. The space vector form of the output voltage and current are expressed as

$$
\begin{gathered}
\boldsymbol{v}_{o}=\frac{3}{2}\left(v_{o a}+\boldsymbol{r} v_{o b}+\boldsymbol{r}^{2} v_{o c}\right) \\
\boldsymbol{i}_{\boldsymbol{o}}=\frac{3}{2}\left(i_{o a}+\boldsymbol{r} i_{o b}+\boldsymbol{r}^{2} i_{o c}\right)
\end{gathered}
$$

where $\boldsymbol{r}=e^{j(2 \pi / 3)}$. The continuous-time model of the load current is defined as

$$
\boldsymbol{v}_{\boldsymbol{o}}=L \frac{d \boldsymbol{i}_{\boldsymbol{o}}}{d t}+R \boldsymbol{i}_{\boldsymbol{o}}
$$

The capacitor voltage dynamic models can be derived as

$$
\begin{aligned}
& C \frac{d v_{c 1}}{d t}=i_{c 1} \\
& C \frac{d v_{c 2}}{d t}=i_{c 2}
\end{aligned}
$$

To acquire the discrete-time model of the control variable, a numerical method can be applied. In this study, the Forward Euler method is used to convert the continuous data to sampled data. The Forward Euler approximation is as follows:

$$
\frac{d f(t)}{d t} \approx \frac{f(k+1)-f(k)}{T_{s}}
$$

$k$ denotes the sampling instant. $T_{s}$ implies the sampling period. By using (6), the discrete-time model of (3) results

$$
\boldsymbol{i}_{\boldsymbol{o}}(k+1)=\left(1-\frac{R T_{s}}{L}\right) \boldsymbol{i}_{\boldsymbol{o}}(k)+\frac{T_{s}}{L} \boldsymbol{v}_{\boldsymbol{o}}(k)
$$

In (7), $\boldsymbol{i}_{\boldsymbol{o}}(k+1)$ is the prediction of the load current, $\boldsymbol{i}_{\boldsymbol{o}}(k)$ refers to the instantaneous load current measurement, and $\boldsymbol{v}_{\boldsymbol{o}}(k)$ denotes the output load voltage. By applying the same procedure, the capacitor voltage prediction models are defined as

$$
\begin{aligned}
& v_{c 1}(k+1)=v_{c 1}(k)+\frac{1}{C_{1}} i_{c 1}(k) T_{s} \\
& v_{c 2}(k+1)=v_{c 2}(k)+\frac{1}{C_{2}} i_{c 2}(k) T_{s}
\end{aligned}
$$


In (8)-(9), $i_{c 1}(k)$ and $i_{c 2}(k)$ are the capacitor current measurements. $C_{1}$ and $C_{2}$ are the capacitance at the inverter input. However, the measurement of these quantities is not a straightforward task (in most cases, the capacitor current measurement is meaningless due to the fast dynamic), $i_{c 1}(k)$ and $i_{c 2}(k)$ can be estimated by using the dynamic model of the capacitor and NPC inverter. The estimation can be performed based on (10) and (11).

$$
\begin{aligned}
& i_{c 1}=i_{d c}(k)-G_{1 a} i_{o a}(k)-G_{1 b} i_{o b}(k)-G_{1 c} i_{o c}(k) \\
& i_{c 2}=i_{d c}(k)-G_{2 a} i_{o a}(k)-G_{2 b} i_{o b}(k)-G_{2 c} i_{o c}(k)
\end{aligned}
$$

where

$$
\begin{aligned}
& G_{1 j}= \begin{cases}1, & S_{j}=P \\
0, & S_{j}=N \text { or } 0\end{cases} \\
& G_{2 j}= \begin{cases}1, & S_{j}=N \\
0, & S_{j}=P \text { or } 0\end{cases}
\end{aligned}
$$

with $\mathrm{j} \in\{\mathrm{a}, \mathrm{b}, \mathrm{c}\} . i_{c 1}(k)$ and $i_{c 2}(k)$ relies on the switch positions. Thus, they can be estimated using the instantaneous switch positions. As a final comment, the prediction model of the system is derived, and the MPC uses (7)-(11) to predict the control goals.

\section{PREDICTIVE CONTROL SCHEME}

The predictive controller uses the prediction model of the system to predict the output load currents and the capacitor voltage for each allowable control inputs (27 switching states). The measurements in the MPC control scheme are the output load current, capacitor voltage, dcbus voltage, and dc-bust current. These four measurements are necessary to apply the predictive control routine. Once the prediction of the control variables process is over, the cost function is evaluated for every legitimate state. The designed objective function is defined as

$$
g_{N P C}=g_{1}+g_{2}+g_{3}
$$

where

$$
\begin{aligned}
& g_{1}=\mid i_{o a}^{*}(k+1)-\left.i_{o a}(k+)\right|^{2} \\
&+\left|i_{o b}^{*}(k+1)-i_{o b}(k+)\right|^{2} \\
&+\left|i_{o c}^{*}(k+1)-i_{o c}(k+)\right|^{2} \\
& g_{2}=\Gamma_{v c}\left|v_{c 1}(k+1)-v_{c 2}(k+1)\right| \\
& g_{3}=\Gamma_{s w c}\left(\left|S_{j 1}(k+1)-S_{j 1}(k)\right|+\cdots\right. \\
& \\
&\left.+\left|S_{j 4}(k+1)-S_{j 4}(k)\right|\right)
\end{aligned}
$$

In (14), the term $g_{1}$ is responsible for tracking the output load current trajectory. The load current error terms are introduced in $g_{1}$ to effectively control the output load current. The term $g_{2}$ controls the capacitor voltages; thus, the voltage imbalance problem is solved by the inclusion of $g_{2}$ term. Moreover, $g_{3}$ is responsible for controlling the switching frequency. In power electronics, the switching loss increases with the switching frequency. Thus, controlling the switching frequency is highly desirable to achieve an acceptable conversion efficiency. The predictive control scheme is depicted in Fig. 2. Following Fig. 2, the control variables are controlled by assessing the objective function. The switching state that offers a minimum cost of $g_{N P C}$ is picked and applied to the NPC inverter. This feedback algorithm is repeated at every sampling instant $k$. Therefore, the MPC implementation can be considered as an iterative operation.

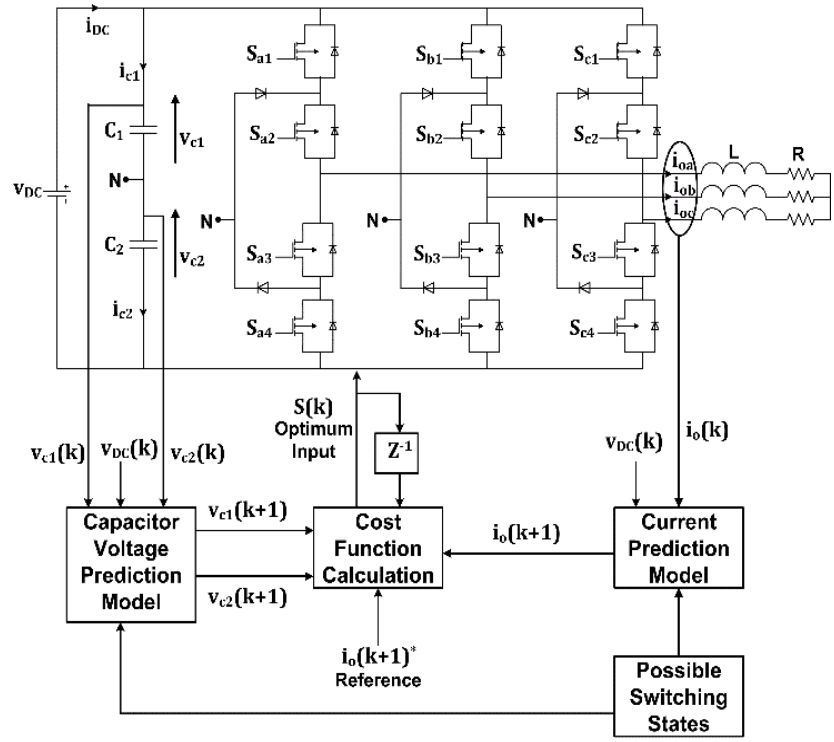

Figure 2. The model predictive control scheme

When $v_{c 1}(k+1)$ and $v_{c 2}(k+1)$ are equal each other, $g_{2}$ results zero. In this case, perfect voltage balancing is achieved. The term $g_{3}$ implies that transition from one state to another is penalized. Thus, $g_{3}$ has a big influence on the switching frequency reduction. The switching state transition rate is inherently limited due to the penalization term of $g_{3}$. The other important design parameters are the weighting factors $\Gamma_{v c}$ and $\Gamma_{s w c}$. $\Gamma_{v c}$ implies the importance of the voltage balancing term. The control of the capacitor voltage equalization becomes more critical as $\Gamma_{v c}$ increases. Thus, the weighting factors are quite important tuning parameters. The weight $\Gamma_{s w c}$ is the tuning parameter of the switching frequency control term $g_{3}$. A large value of $\Gamma_{s w c}$ offers a better switching frequency regulation. In an optimization problem formed by more than one control goal, the weighting factors are required to handle the relationship between control terms. The critical comment on the nomination of these weighting factors is that a good approach to determine the weighting factors has not been proposed yet. In most cases, simulation-based tuning methods are used to determine these tuning parameters.

\section{SIMULATION RESULTS AND PERFORMANCE EVALUATION}

To perform the performance analyses, the system is simulated using Matlab/Simulink. The simulation parameters are given in Table 2. To assess the steadystate performance, the control variables are monitored. Fig. 3 presents the steady-state waveforms. In this test scenario, the load current reference is $10 \mathrm{~A}$ peak with 50 
Hz fundamental frequency. According to the steady-state results, the output load current looks good, see Fig. 3(a). The capacitor balancing performance is acceptable, see Fig. 3(b), no big deviation between $v_{c 1}$ and $v_{c 2}$ is observed.

Table 2. Simulation parameters

\begin{tabular}{lll}
\hline Parameters & Description & Values \\
\hline $\mathrm{V}_{\mathrm{dc}}$ & dc-bus voltage & $380 \mathrm{~V}$ \\
$\mathrm{C}_{1}$ & $\mathrm{C}_{1}$ capacitance & $750 \mu \mathrm{F}$ \\
$\mathrm{C}_{2}$ & $\mathrm{C}_{2}$ capacitance & $750 \mu \mathrm{F}$ \\
$\mathrm{R}$ & Load resistance & $5 \Omega$ \\
$\mathrm{L}$ & Load inductance & $10 \mathrm{mH}$ \\
$\mathrm{T}_{\mathrm{s}}$ & Sampling period & $20 \mu \mathrm{s}$ \\
$\Gamma_{v c}$ & Weighting factor & 0.6 \\
$\Gamma_{s w c}$ & Weighting factor & 0.05 \\
Solver & Simulink solver & Ode-45 \\
\hline
\end{tabular}

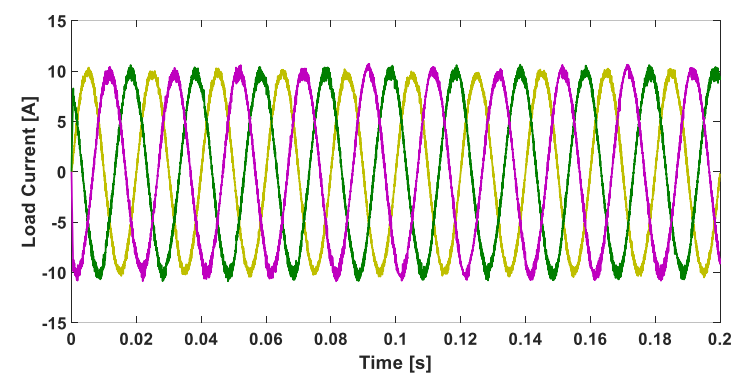

(a)

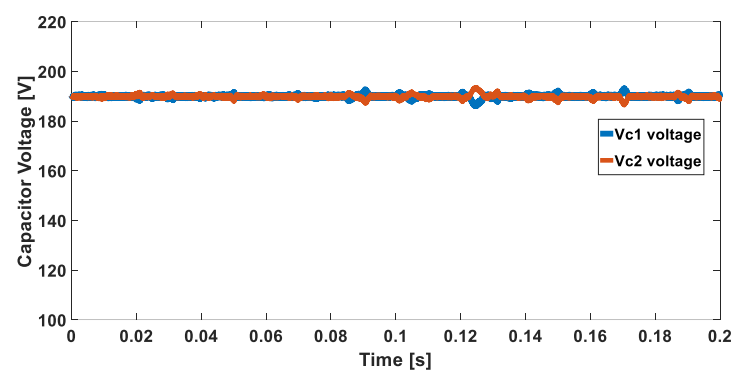

(b)

Figure 3. The steady-state waveform of the predictive controller. (a) The output load current waveform (b) Capacitor voltage waveform.

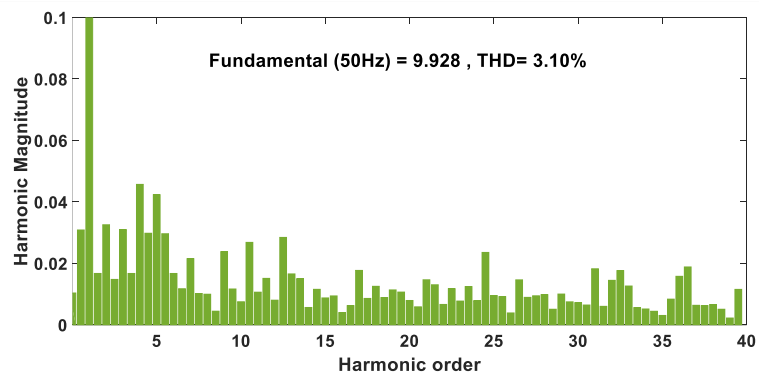

Figure 4. FFT analysis of the load current up to $40^{\text {th }}$ harmonics.

To examine the load current quality, Fast Fourier Transform (FFT) analysis is conducted. The spectral contents are calculated up to $40^{\text {th }}$ harmonics. The frequency spectrum waveform of the output load current is reported in Fig. 4 under steady-state conditions. The load current total harmonic distortions are roughly $3.10 \%$. The calculated THD level is quite acceptable, and the quality of the load current satisfies the design specifications.

The magnitude of the fundamental frequency $(50 \mathrm{~Hz})$ is $9.928 \mathrm{~A}$, close to the reference value of $10 \mathrm{~A}$. To investigate the transient performance of the predictive controller, the load current step is applied to the NPC inverter system. The transient response of the system is reported in Fig. 5. The load current step is applied to $5 \mathrm{~A}$ peak to $10 \mathrm{~A}$. The predictive controller gives a quick response to the load variations and compensates for the error. The capacitor voltage control is attained, no unwanted discrepancy is observed. Both capacitor voltage is approximately $190 \mathrm{~V}$ which corresponds to half of the dc-bus voltage VDC.

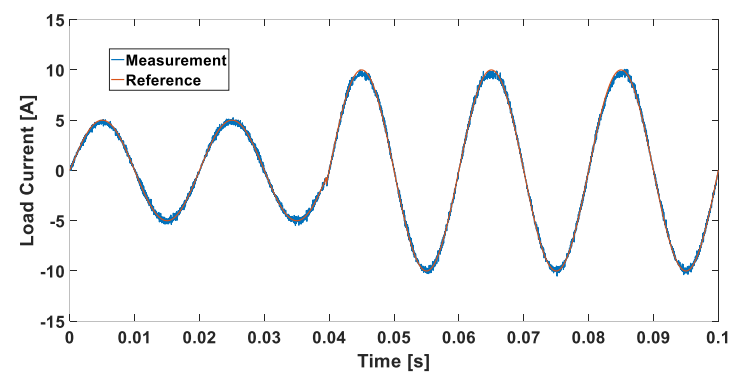

(a)

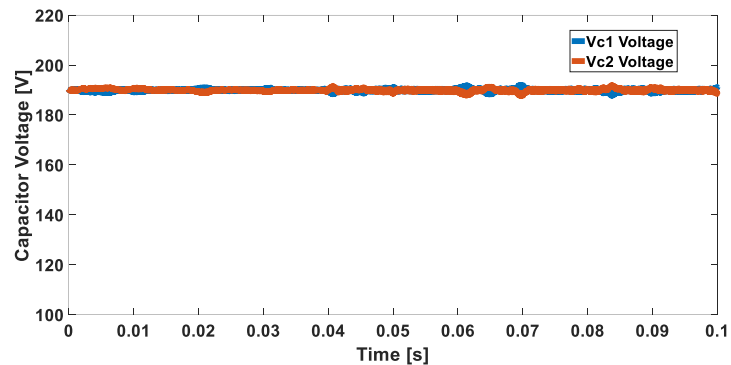

(b)

Figure 5. The transient waveform of the predictive controller. (a) The load current waveform (measurement and reference) (b) Capacitor voltage waveform

To explore the weighting factor $\Gamma_{v c}$ effects on the capacitor voltage balancing performance, $\Gamma_{v c}$ set as 0 . Then, $\Gamma_{v c}$ has switched to its nominal value of 0.6 . When $\Gamma_{v c}=0$, the capacitor voltage balancing is ignored by the predictive controller. It means that the capacitor voltage imbalance is not penalized; thus, the capacitor voltages may not be equal. The effect of $\Gamma_{v c}$ is presented in Fig. 6, and the fine choice of $\Gamma_{v c}$ remarkably reduces the voltage imbalance. These simulation results prove that the predictive controller performance highly relies on the selected weighting factors.

For $\Gamma_{v c}=0, v_{c 2}$ is approximately $200 \mathrm{~V}$ while $v_{c 1}$ is 180 $\mathrm{V}$ at the same time. This causes voltage imbalance. For $\Gamma_{v c}=0.6$, both capacitor voltages approximately equal 190 $\mathrm{V}$. 


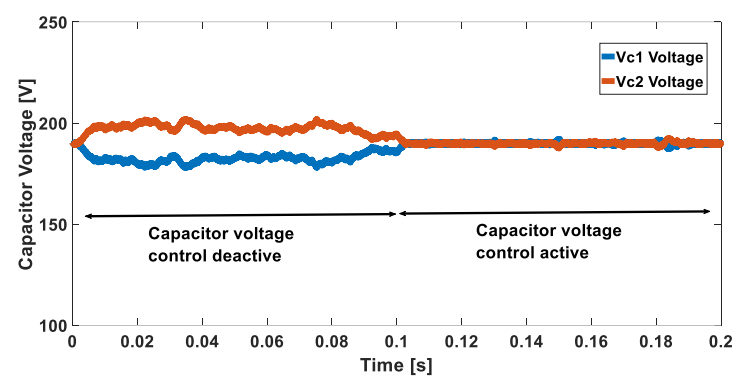

Figure 6. The impacts of the parameter $\Gamma_{v c}$ on the term $\left|v_{c 1}-v_{c 2}\right|$.

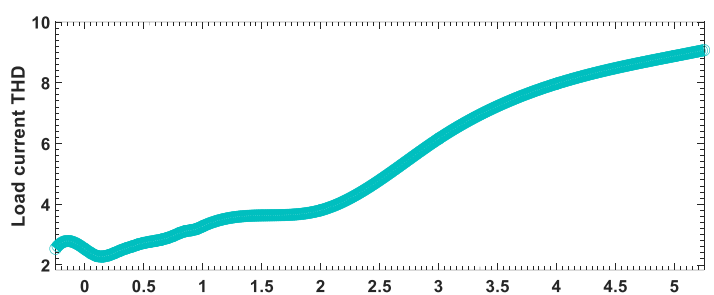

(a)

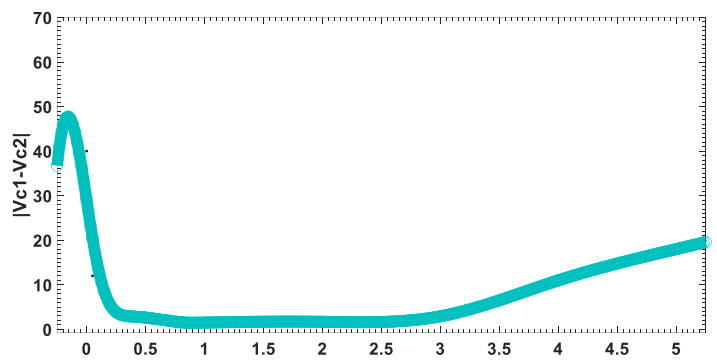

(b)

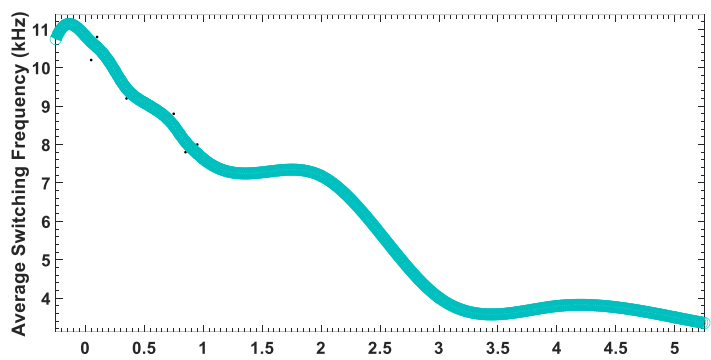

(c)

Figure 7. The impacts of the parameter $\Gamma_{v c}$ on the control variables. (a) The waveform $\Gamma_{v c}$ vs load current THD. (b) The waveform $\Gamma_{v c}$ vs. $\left|v_{c 1}-v_{c 2}\right|$. (c) The waveform $\Gamma_{v c}$ vs. operating frequency.

The effects of the parameter $\Gamma_{v c}$ on the controller, variables are shown in Fig. 7. The load current has a high THD tendency as $\Gamma_{v c}$ increases. For the lower choice of $\Gamma_{v c}$ such as $0.05<\Gamma_{v c}<0.8$, the load current THD varies between $2.43 \%<\mathrm{THD}<3.1 \%$. This THD level is quite acceptable, and it complies with the international standard. However, further, increase in $\Gamma_{v c}$ degrade the load current quality, see Fig. 7 (a). The choice of $\Gamma_{v c}>2$ has a negative influence on the load current THD. The THD level is drastically increased; thus, the selection of $\Gamma_{v c}$ is quite critical. The capacitor voltage imbalance is reduced with a higher value of $\Gamma_{v c}$. The choice of $0.05<\Gamma_{v c}<3$ improves the capacitor voltage control, the parameter $\left|v_{c 1}-v_{c 2}\right|$ varies between $6 \mathrm{~V}$ and $2.9 \mathrm{~V}$. However, the further increase in $\Gamma_{v c}$ does not help to improve the control of $\left|v_{c 1}-v_{c 2}\right|$. The voltage imbalance equals $18 \mathrm{~V}$ when $\Gamma_{v c}$ is selected as 5 . The main reason for the practical limit on the capacitor voltage control performance is that a higher value of $\Gamma_{v c}$ causes the increase in the load current THD. Therefore, the control of the capacitor voltage becomes challenging due to the high distortions. For that reason, $\Gamma_{v c}$ should be selected such that all control variables remain in the acceptable range. The switching frequency decreases with the increase in $\Gamma_{v c}$. Fig. 7 (c) show the mean value of the operating frequency versus the $\Gamma_{v c}$. The computed average operating frequency range is within $3.5 \mathrm{kHz}<f_{s w}<11 \mathrm{kHz}$. By taking into all analyses results presented in Fig.7, the range within $0.05<\Gamma_{v c}<1$ is the sweet spot for $\Gamma_{v c}$. The selection of $\Gamma_{v c}$ within this range offers a good control performance of all control goals. The last evaluation work is performed to analyze the effects of switching frequency control term $\Gamma_{s w c}$. In this performance assessment process, two control variables are under consideration: the load current THD and the average switching frequency. The effect of $\Gamma_{s w c}$ on the load current, THD, and the mean value of the operating frequency is shown in Fig. 8. The load current THD increases as the value of $\Gamma_{s w c}$ increases. In particular, the selection of $\Gamma_{s w c}>4$ degrades the output load current control performance. The key reason for this harmful effect is that the switching frequency is noticeably reduced with the increase in $\Gamma_{s w c}$, see Fig. 8 (b). The switching transitions are further penalized by increasing $\Gamma_{s w c}$. Thus, the NPC inverter does fewer switching transitions resulting in a lower switching frequency. However, the output load current control is negatively influenced. Based on the collected simulation results, the selection of $0.5<\Gamma_{s w c}<2.5$ is recommended to attain good steady-state performance.

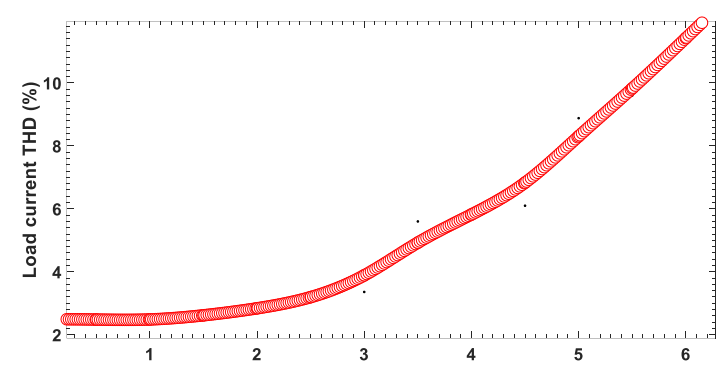

(a)

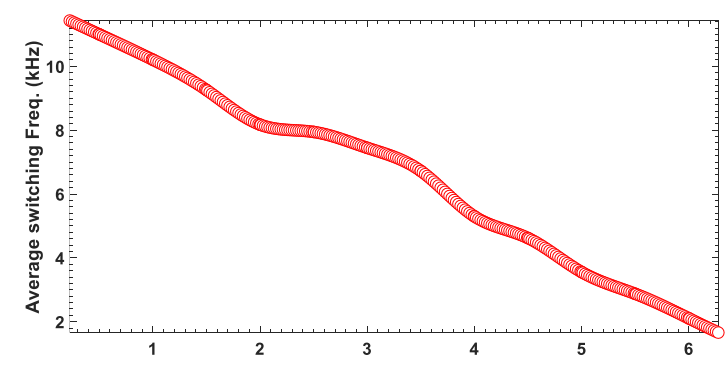

(b)

Figure 8. The impact of the tuning parameter $\Gamma_{s w c}$ on the control variables. (a) The waveform $\Gamma_{s w c}$ vs. load current THD. (b) The waveform $\Gamma_{s w c}$ vs. switching frequency.

Finally, the proposed control method is compared with the conventional control technique which is based on the proportional-integral (PI) combined with the 
linear modulator. Table 3 summarizes the comparison results. According to Table 3, the proposed method offers faster dynamic response than the conventional method to load perturbations. The transient time is noticeably lower for the proposed strategy. On the other hand, the traditional method provides a lower THD under steadystate performance.

Table 3. Comparison results

\begin{tabular}{lll}
\hline Metric & PI+Modulator & MPC \\
\hline Transient time & $210 \mu \mathrm{s}$ & $155 \mu \mathrm{s}$ \\
Load current THD & $3.05 \%$ & $3.10 \%$ \\
Modulator & Required & No \\
Inclusion of constraints & No & Yes \\
Inclusion of nonlinearity & No & Yes \\
Switching frequency & Fixed & Variable \\
Design complexity & High & Low \\
Voltage balancing & Yes & Yes \\
\hline
\end{tabular}

The PI+modulator requires the linear modulator to generate the gate signals. Thus, the switching frequency is fixed. However, the inclusion of the modulator increases the complexity of the design stage. On the contrary, the MPC strategy does not require a modulator; therefore, the switching frequency varies during the operation. Both control methods offer voltage balancing capability. The other important aspect is that the conventional method does not provide the flexibility to include the nonlinearities or control constraints. On the other hand, the inclusion of the system constraints is relatively easy in the MPC method. Regarding the simplicity of the feedback design, the MPC method has several advantages over the traditional control method.

\section{CONCLUSION}

This paper has presented the performance evaluation of the predictive control method for the NPC inverter. The performance analyses have been conducted to scrutinize the steady-state performance and transient performance of the MPC. The impacts of the design parameters on the trajectory tracking performance have been substantially analyzed. Several simulation works have been conducted to prove the effectiveness of the MPC in handling the multi-control objectives. The simulation results demonstrate that multiple-control goals are effectively regulated, and the NPC inverter provides a robust system operation. The choice of the weighting factor highly influences the error compensation performance. Thus, weighting factors should be finely selected to achieve a reliable energy conversion operation.

\section{Author contributions}

Ozan Gulbudak: Design and analyses, Methodology Mustafa Gokdag: Simulation, WritingOriginal draft preparation.

\section{Conflict of interest}

The authors declare no conflicts of interest

\section{REFERENCES}

Choi U-M, Blaabjerg F \& Lee K-B (2015). Method to Minimize the Low-Frequency Neutral-Point Voltage Oscillations With Time-Offset Injection for NeutralPoint-Clamped Inverters. IEEE Transactions on Industry Applications, 51(2), 1678-1691. https://doi.org/10.1109/TIA.2014.2350079

Jarutus N \& Kumsuwan Y (2017). A Carrier-Based PhaseShift Space Vector Modulation Strategy for a NineSwitch Inverter. IEEE Transactions on Power Electronics, 32(5), 3425-3441. https://doi.org/10.1109/TPEL.2016.2587811

Jie S, Schröder S, Rösner R \& El-Barbari S (2011). A Comprehensive Study of Neutral-Point Self-Balancing Effect in Neutral-Point-Clamped Three-Level Inverters. IEEE Transactions on Power Electronics, 26(11), 3084-3095. https://doi.org/10.1109/TPEL.2011.2138161

Klabunde M C, Yifan Z, \& Lipo T A (1994). Current control of a 3-level rectifier/inverter drive system. Proceedings of 1994 IEEE Industry Applications Society Annual Meeting, 859-866. https://doi.org/10.1109/IAS.1994.377519

Nabae A, Takahashi I \& Akagi H (1981). A New NeutralPoint-Clamped PWM Inverter. IEEE Transactions on Industry Applications, IA-17(5), 518-523. https://doi.org/10.1109/TIA.1981.4503992

Newton C \& Sumner M (1997). Neutral point control for multi-level inverters: theory, design and operational limitations. IAS '97. Conference Record of the 1997 IEEE Industry Applications Conference Thirty-Second IAS Annual Meeting, 2, 1336-1343. https://doi.org/10.1109/IAS.1997.629031

Ogasawara S \& Akagi H (1993). Analysis of variation of neutral point potential in neutral-point-clamped voltage source PWM inverters. Conference Record of the 1993 IEEE Industry Applications Conference Twenty-Eighth IAS Annual Meeting, 965-970. https://doi.org/10.1109/IAS.1993.299015

Rodriguez J, Bernet S, Steimer P K \& Lizama I E (2010). A Survey on Neutral-Point-Clamped Inverters. IEEE Transactions on Industrial Electronics, 57(7), 22192230. https://doi.org/10.1109/TIE.2009.2032430

Rojas R, Ohnishi T \& Suzuki T (1995). An improved voltage vector control method for neutral-pointclamped inverters. IEEE Transactions on Power Electronics, 10(6), 666-672. https://doi.org/10.1109/63.471286

Steinke J K (1992). Switching frequency optimal PWM control of a three-level inverter. IEEE Transactions on Power Electronics, 7(3), 487-496. https://doi.org/10.1109/63.145136

Vargas R, Cortes P, Ammann U, Rodriguez J \& Pontt J (2007). Predictive Control of a Three-Phase NeutralPoint-Clamped Inverter. IEEE Transactions on Industrial Electronics, 54(5), 2697-2705. https://doi.org/10.1109/TIE.2007.899854 